Supporting Information for

\title{
Simultaneous Real-Time Three-Dimensional Localization and FRET Measurement of Two Distinct Particles
}

\author{
Xingxiang Chen ${ }^{1, \S}$, Teng Liu ${ }^{2, \S}$, Xianan Qin" ${ }^{2,}$,\#, Quang Quan Nguyen², Sang Kwon Lee ${ }^{3}$, \\ Chanwoo Lee ${ }^{4}$, Yaguang Ren ${ }^{5}$, Jun $\mathrm{Chu}^{6}$, Guang Zhu',5, Tae-Young Yoon ${ }^{4}$, Chan Young \\ Park $^{3}$ and Hyokeun Park ${ }^{1,2,7, *}$
}

${ }^{1}$ Division of Life Science, The Hong Kong University of Science and Technology, Clear Water Bay, Kowloon, Hong Kong

${ }^{2}$ Department of Physics, The Hong Kong University of Science and Technology, Clear Water Bay, Kowloon, Hong Kong

${ }^{3}$ Department of Biological Sciences, School of Life Sciences, UNIST, 44919, Ulsan, Republic of Korea

${ }^{4}$ School of Biological Sciences and Institute of Molecular Biology and Genetics, Seoul National University, Seoul 08826, South Korea

${ }^{5}$ Department of Chemical and Biological Engineering, The Hong Kong University of Science and Technology, Clear Water Bay, Kowloon, Hong Kong

${ }^{6}$ Research Lab for Biomedical Optics and Molecular Imaging, Shenzhen Institutes of Advanced Technology, Chinese Academy of Sciences, Shenzhen, 518055, China

${ }^{7}$ State Key Laboratory of Molecular Neuroscience

The Hong Kong University of Science and Technology, Clear Water Bay, Kowloon, Hong Kong

${ }^{\S}$ X.C., T.L. and X.Q. contributed equally to this work.

\# Current address: National Engineering Lab for Textile Fiber Materials \& Processing Technology, College of Textile Science and Engineering, Zhejiang Sci-Tech University, Hangzhou 310018, China

*Corresponding author: Hyokeun Park

E-mail: hkpark@ust.hk 


\section{Materials and methods}

\section{Oligonucleotides for single-molecule FRET experiments}

The following oligonucleotides of sense and antisense sequences (all written from 5' end to 3' end) were purchased from IDTDNA (Integrated DNA Technologies, Inc., USA): Sense Sequence:

Biotin-5'-CC AGA CAA ACA CTC AAA CAA ACT CGA CAC TTT CAG CTC-3'ATTO647N

Antisense sequence:

ATTO550-5'- GAG CTG AAA GTG TCG AGT TTG TTT GAG TGT TTG TCT GG - 3'

\section{Plasmids and primers}

For mRuby3-2aa-mClover3, mRuby3 with BamHI site and mClover3 with BamHI/BglII sites were amplified using PCR with the primer sets shown below and cloned into pCR8/GW/TOPO cloning vector (Invitrogen) by TA cloning. pCR8-BamHI-mClover3BglII was digested with BamHI/BglII to yield the insert BamHI-mClover3-BglII. After pCR8mRuby3-BamHI was cleaved by BamHI, the insert BamHI-mClover3-BglII was cloned into pCR8-mRuby3-BamHI with T4 DNA ligase (New England Biolabs), generating pCR8mRuby3-BamHI-mClover3. pCR8-mRuby3-BamHI-mClover3 was transferred into the pDEST expression vector by Gateway system (Gateway LR Clonase II; Invitrogen, Carlsbad, CA) to generate mRuby3-2aa-mClover3.

For mRuby3-80aa-mClover3, a tandem repeat of ELP (Elastin Like Polypeptide) was first generated by annealing both primers with BglII and BamHI sites at both ends (BglII(VPGVG)X4-BamHI, ELP20) and cloned into a custom made pCY3 vector (based on pCR8/GW/TOPO vector) containing BglII and BamHI sites. pCY3-BglII-ELP20-BamHI, which contains XbaI site behind inserted annealed oligomer, was digested with BamHI/XbaI and BglII/XbaI and separated into the vectors (pCY3-BglII-ELP20-BamHI-(sticky ends)$\mathrm{XbaI}$ ) and inserts (BglII-ELP20-BamHI-XbaI), respectively. After two repeat of subcloning, the vector (pCY3-BglII-ELP20::ELP20::ELP20::ELP20-BamHI-XbaI, containing 80 aa of ELP repeats) was generated and then digested with BamHI/BglII to isolate the insert. Finally, the insert Bg1II-ELP20::ELP20::ELP20::ELP20-BamHI was subcloned into BamHI site of mRuby3-2aa(BamHI)-mClover3 to generate mRuby3-80aa-mClover3.

The following primer sets were used.

For ELP (Elastin Like Polypeptide, cleavaged BglII-ELPx4-BamHI): 
Forward 5'-

GATCTGTTCCTGGTGTTGGGGTACCAGGAGTAGGCGTCCCCGGCGTCGGAGTGCC GGGGGTGGGTG-3'

Reverse 5'-

GATCCACCCACCCCCGGCACTCCGACGCCGGGGACGCCTACTCCTGGTACCCCAA CACCAGGAACA-3'

For mClover3 (BamHI-mClover3-BgIII):

Forward 5'-GGATCCATGGTGAGCAAGGGCGAG-3'

Reverse 5'-AGATCTCTACTTGTACAGCTCGTCCATGCCA-3'

For mRuby3 (mRuby3-BamHI):

Forward 5'-GGTACCGGTTCAGGCATGGTGTCTAAGGGCGAAGAGCTG-3'

Reverse 5'-GGATCCCTTGTACAGCTCGTCCATGCCA-3'

\section{Cell culture and transfection}

HEK293T cells were cultured in DMEM (Thermo Fisher Scientific) supplemented with 10\% (v/v) FBS (Thermo Fisher Scientific). To perform fluorescence imaging experiments, cells at $\sim 70-80 \%$ confluence in $6 \mathrm{~cm}$ culture dishes were transfected with plasmids encoding mRuby3-2aa-mClover3 and mRuby3-80aa-mClover3 using Lipofectamine 2000 (Invitrogen). Culture media was changed 4-5 hours after transfection, and the cells were split and seeded onto poly-D-Lysine (Sigma-Aldrich) coated coverslips in the following day. Fluorescence images were taken 48-72 hours after transfection. ${ }^{1,2}$

\section{Microspheres sample preparation}

A stock solution of TetraSpeck microspheres with a diameter of $0.1 \mu \mathrm{m}$ (T7279, Thermo Fisher Scientific) was diluted 100 times with the same imaging buffer as singlemolecule FRET experiment or single-vesicle fusion assay. To immobilize these microspheres on the coverslips, $1 \mu \mathrm{L}$ of $1 \mathrm{mM} \mathrm{HCl}$ solution was added to $100 \mu \mathrm{L}$ of the diluted microspheres solution. This mixture was then loaded into a custom-built chamber and incubated at room temperature for 15 minutes to allow these microspheres to adhere to cover glasses. ${ }^{3,4}$ The custom-built chamber was made as previously described. ${ }^{3,5}$ 


\section{Fluorescence imaging experiments of microspheres}

Imaging experiments were performed using an IX73 inverted fluorescence microscope (Olympus) equipped with an oil-immersion objective (NA $=1.49,100 \times$, UAPON, Olympus). A 532-nm laser (Coherent Inc., USA) or a 640-nm laser (Coherent Inc.) was used to excite the fluorescent microspheres alternatingly. A dichroic mirror (ZT532/640rpc, Chroma) was used to collect the emission signals, which were later divided into two spectral distinct channels by a second dichroic mirror (T6471pxr, Chroma) mounted near the side-port of the microscope and filtered by ET580/60m (Chroma) and ET690/50m (Chroma), respectively. The two emission signals were finally collected and focused onto an iXon Ultra EM-CCD camera (Andor, UK).

A PIFOC Objective Scanner (P-725, Physik Instrumente) was used to generate $100 \mathrm{~nm}$ steps along the z-axis. For each step, 100 frames images with $100 \mathrm{~ms}$ exposure time were taken under the alternating illumination of $532 \mathrm{~nm}$ and $640 \mathrm{~nm}$ lasers and separated into two spectrally distinct channels. Fluorescent images of microspheres were fitted with 2D Gaussian functions to calculate widths of PSFs along the $\mathrm{x}$-and $\mathrm{y}$-axis ( $w_{x}$ and $w_{y}$ ) by using custom-made IDL programs (L3Harris Geospatial, USA). Calibration curves based on the widths ( $w_{x}$ and $w_{y}$ ) or ellipticity $\left(w_{x} / w_{y}\right)$ of PSFs as a function of $\mathrm{z}$ position were used to determine the positions along the z-axis.

\section{Mapping 3D localizations of fluorophores in the two channels}

A novel algorithm was developed for accurately transforming 3D localizations from one imaging channel to the other imaging channel. The algorithm is based on the idea of "local weighted mean" (LWM), which was originally proposed for 2D imaging registration by Goshtasby ${ }^{6}$, and later used for the transformation problem of 2D single-molecule localization by Spudich's group. ${ }^{7}$ The 3D transformation can be formulated as finding a mathematical model which is able to accurately transform 3D coordinates of fluorophores in one channel $\left(x_{i 1}, y_{i 1}, z_{i 1}\right)$ to the other channel $\left(x_{i 2}, y_{i 2}, z_{i 2}\right)$ where $i$ denotes the $i$-th fluorophore. Similar to Goshtasby's method, our algorithm first generates all polynomial features of the coordinates in one channel with degree less than or equal to 2 :

$$
p_{i}=\left(1, x_{i 1}, y_{i 1}, z_{i 1}, x_{i 1} y_{i 1}, x_{i 1} z_{i 1}, y_{i 1} z_{i 1}, x_{i 1}{ }^{2}, y_{i 1}{ }^{2}, z_{i 1}{ }^{2}\right)
$$

The algorithm locates $N$ nearest fluorophores in the neighbored 3D space for each localization. Following the default setting as for 2D LWM algorithms in MATLAB (for example, the 'lwm' option of the cp2tform function), $N$ is by default set as 12 . Then, the 
algorithm fits multivariate linear regression models $f_{i}\left(x_{1}, y_{1}, z_{1}\right)$ of which the inputs and outputs are the polynomial features $p_{i}$ and the transformed coordinates $x_{i 2}, y_{i 2}, z_{i 2}$, respectively. The gross mapping model of our algorithm is made up of $K$ individual regression models if there are $K$ pairs of coordinates used in the training dataset. When the gross mapping model is used for transforming localizations outside the training data, our algorithm automatically finds 13 nearest localizations in the training dataset and generates a weighted polynomial model for transforming the localizations. The weights are calculated based on the following rules ${ }^{6}$ :

$$
W_{j}=\left\{\begin{array}{c}
1-3 R_{j}^{2}+2 R_{j}{ }^{3}, 0 \leq R_{j} \leq 1 \\
0, \text { otherwise }
\end{array}\right.
$$

where $R_{j}$ is the $j$-th distance ratio and is defined and calculated as:

$$
R_{j}=\frac{\sqrt{\left(x_{1}-x_{j 1}\right)^{2}+\left(y_{1}-y_{j 1}\right)^{2}+\left(z_{1}-z_{j 1}\right)^{2}}}{d_{n}}
$$

where $x_{j 1}, y_{j 1}$ and $z_{j 1}$ are the $j$-th set of coordinates in the channel 1 of the 13 nearest localizations; $x_{1}, y_{1}$ and $z_{1}$ are the coordinates in the channel 1 , which are to be registered with the coordinates in the channel 2 ; and $d_{n}$ denotes the distance from $\left(x_{1}, y_{1}, z_{1}\right)$ to the farthest coordinate of the 13 nearest localizations. For each $R_{j}$, there is a multivariate linear regression model that has been generated in the generation of the gross mapping model. Our algorithm finally generates a weighted polynomial function for each coordinate:

$$
F\left(x_{1}, y_{1}, z_{1}\right)=\frac{\sum_{j} W_{j} f_{j}\left(x_{1}, y_{1}, z_{1}\right)}{\sum_{j} W_{j}}
$$

which transforms $\left(x_{1}, y_{1}, z_{1}\right)$ to $\left(x_{2}, y_{2}, z_{2}\right)$.

We used total 200 pairs of 3D localizations of beads to generate the gross mapping model, and calculated the fiducial registration error (FRE). ${ }^{7}$ The calculated FRE in $\mathrm{x}, \mathrm{y}$ and $\mathrm{z}$ directions is used to determine whether our transformation algorithm accurately maps locations of single fluorophores in three dimensions.

\section{Single-molecule FRET experiments}

Sample chambers were prepared as previously described. ${ }^{8}$ PEGylation was performed with freshly prepared PEG solution (for 1 slide: $10 \mathrm{mg}$ mPEG-SVA-5000 (Methoxypolyethylene glycol-succinimidyl valerate, Laysan Bio) and $0.25 \mathrm{mg}$ Biotin-PEG-SVA-5000 (Biotin-polyethylene glycol-succinimidyl valerate, Laysan Bio) in $40 \mu \mathrm{L} 0.1 \mathrm{M}$ fresh sodium bicarbonate buffer, $\mathrm{pH} 8.5$ ), which was filtered through a $0.45 \mu \mathrm{m}$ filter and centrifuged at $10000 \mathrm{x}$ g for 1 minute. We briefly added $40 \mu \mathrm{L}$ PEG solution between a dried cover glass and 
a dried slide to form a sandwich structure and incubated at a humid and dark environment for 3 hours. We disassembled and rinsed PEGylated glasses several times with Milli-Q water and dried them with nitrogen gas. Then, a sample chamber was assembled with a pair of PEGylated coverslip and slide using double-sided sticky and Epoxy glue. Sample chambers were stored at $-20^{\circ} \mathrm{C}$.

Two single-stranded oligonucleotides were dissolved in annealing buffer (10 mM Tris $\mathrm{pH} 7.5,50 \mathrm{mM} \mathrm{NaCl}$ and $1 \mathrm{mM}$ EDTA). Equal volumes of the equimolar oligonucleotides were mixed thoroughly. The mixture was incubated in a preheated heat block at $95{ }^{\circ} \mathrm{C}$ for 2 minutes and allowed to cool down to room temperature slowly.

To immobilize double-stranded DNA molecules on the chamber channel surface, 0.2 $\mathrm{mg} / \mathrm{ml}$ Neutravidin (31050, Thermo Scientific) diluted in Tris buffer (10 mM Tris- $\mathrm{HCl}, 50 \mathrm{mM}$ $\mathrm{NaCl}$ ) was applied into PEGylated chamber for 10 minutes. Excess Neutravidin was gently washed out before $0.1 \%$ BSA blocking. Double-strand DNA molecule $(\sim 5 \mathrm{pM})$ was later loaded into the sample chamber and incubated for $5 \mathrm{mins}$ at room temperature. Unbounded DNA molecules were washed out with Tris buffer. Single-molecule FRET experiments were performed in enzymatic oxygen-scavenging imaging buffer $(0.56 \mathrm{mg} / \mathrm{mL}$ glucose oxidase, $0.034 \mathrm{mg} / \mathrm{mL}$ catalase $10 \%$ glucose, $10 \mathrm{mM} \mathrm{NaCl}, 50 \mathrm{mM}$ Tris, $\mathrm{pH} 8.0$ ) with addition of $2 \mathrm{mM}$ Trolox and $10 \mathrm{mM}$ MEA. The abovementioned imaging setup was used for experiments. Double-stranded DNA molecules were illuminated by a 532-nm laser (Coherent Inc.). Objective-based total internal reflection (TIR) illumination was applied to decrease background signals. Fluorescence signals were divided into two spectral channels by a dichroic mirror (T647lpxr, Chroma), and focused to the EMCCD. An emission filter (ET580/60m, Chroma) was used to collect the fluorescence signals of ATTO 550 in the donor channel and another emission filter (ET690/50m, Chroma) was used to collect the fluorescence signals of ATTO $647 \mathrm{~N}$ in the acceptor channel. The fluorescence intensities of the donor $\left(I_{D D}\right)$ and the acceptor $\left(I_{D A}\right)$ channels under the 532-nm laser illumination were calculated using MetaMorph software (Molecular Device) using the same size of region of interest (ROI) with 7-pixel in diameter. The FRET efficiency was calculated using following equation ${ }^{9,10}$ :

$$
E=\frac{I_{D A}}{I_{D D}+I_{D A}}
$$

\section{Live-cell FRET measurement using a fluorescent-protein based FRET pair in living HEK293T cells}

All imaging experiments were performed at room temperature using an Olympus IX73 inverted microscope. 488-nm and 561-nm lasers (Coherent) were used to excite mClover3 
and mRuby3, respectively. A dichroic mirror (ZT488/561rpc-UF1, Chroma) and an emission filter (ET488/561m, Chroma) were used to collect fluorescence signals. An additional dichroic mirror (T5351pxr, Chroma) was used to split the green and red fluorescence from the emission signals. For FRET measurements, alternating laser illumination was applied to acquire the donor signal under donor excitation $\left(I_{D D}\right)$, the FRET signal $\left(I_{D A}\right)$, and the acceptor signal under acceptor excitation $\left(I_{A A}\right)$, respectively. Live-cell FRET efficiency was calculated as ${ }^{1,11-13}$

$$
E_{F R E T}=\left(I_{D A}-a \times I_{D D}-b \times I_{A A}\right) / I_{A A}
$$

in which $a$ is a bleeding-through correction factor of the donor emission in the acceptor channel and $b$ is a direct excitation correction factor of the acceptor fluorophore (mRuby 3 ) by a donor excitation laser. All intensities were measured using MetaMorph.

\section{Single-vesicle fusion assay}

SNAREs reconstituted vesicles were prepared as previously described. ${ }^{14}$ Firstly, the Avanti lipids (Avanti Polar Lipids) including DOPS (1,2-Dioleoyl-sn-glycero-3-phospho-1serine, 840035), POPC (1-Palmitoyl-2-oleoyl-sn-glycero-3-phospho-choline, 850457), DOPE (1,2-Dioleoyl-sn-glycerol-3-phosphoethanolamine, 850725) and Biotinyl Cap PE (1,2Dipalmitoyl-sn-glycero-3-phosphoethanolamine-N-(cap biotinyl), 870277) were dissolved in chloroform. Cholesterol (372978, Sigma-Aldrich) was dissolved in a 19:1 v/v mixture of chloroform: methanol. DiD (1,1'-Dioctadecyl-3,3,3',3'-tetramethylindodicarbocyanine perchlorate, D307, Invitrogen) and DiI (1,1'-Dioctadecyl-3,3,3',3'tetramethylindocarbocyanine perchlorate, D282, Invitrogen) were dissolved in ethanol. We mixed them to 1.5 micro moles lipid mixtures. The DiI contained lipid mixture was composed of 15.0 mole \% DOPS, 44.3 mole \% POPC, 20.0 mole \% DOPE, 18.0 mole \% Cholesterol, 0.7 mole \% Biotinyl Cap PE and 2.0 mole \% DII. The DiD contained lipid mixture consisted of 15.0 mole \% DOPS, 55.0 mole \% POPC, 20.0 mole \% DOPE, 8.0 mole \% Cholesterol, and 2.0 mole \% DiD. Lipid mixtures were evaporated with nitrogen gas and further dried in a vacuum desiccator for at least 3 hours to remove organic solvents. Dried lipids containing DiI and DiD were reconstituted in HEPES buffer (50 mM HEPES, $150 \mathrm{mM} \mathrm{NaCl}, \mathrm{pH} 7.4$ ) to a final lipid concentration of $15 \mathrm{mM}$ with a total of $2 \%$ OG (N-Octyl- $\beta$-d-glucopyranoside, N02007, RPI) and t-SNARE and v-SNARE proteins at a desired ratio, respectively. The mixtures were rocked at $4{ }^{\circ} \mathrm{C}$ for 30 minutes, diluted 3 times with HEPES buffer, and then dialyzed with $10 \mathrm{~K}$ MWCO Slide-A-LyzerTM MINI Dialysis device (88401, Thermo Scientific) overnight in SM-2 
adsorbents (1523920, Bio-Rad) contained HEPES dialysis buffer. Vesicles were finally harvested for single-vesicle fusion experiments.

To immobilize t-vesicles on the chamber channel surface, $0.2 \mathrm{mg} / \mathrm{ml}$ Neutravidin (31050, Thermo Scientific) diluted in Tris buffer (10mM Tris- $\mathrm{HCl}, 50 \mathrm{mM} \mathrm{NaCl})$ was injected into the chamber and mounted to PEGylated surface for 10 minutes. Excess Neutravidin was gently washed out with HEPES buffer. Nonspecific binding was minimized on the surface using $0.1 \%$ BSA solution for 15 minutes. $20 \mu \mathrm{L}$ t-vesicles (final concentration of $3 \mu \mathrm{M}$ ) were loaded into the chamber and incubated for 15 minutes to allow biotinylated t-vesicles to anchor to surface-mounted Neutravidin. We washed out excess t-vesicles with HEPES buffer. Once $\mathrm{v}$-vesicles flowed into chamber, we performed the imaging experiment using Olympus IX-73 inverted fluorescence microscope as abovementioned. DiI contained t-vesicles (donor) and DiD-contained t-vesicles (acceptor) were illuminated by alternate excitation of 532-nm and 640-nm lasers (Coherent) with an exposure time of $100 \mathrm{~ms}$. A dichroic mirror (ZT532/640rpc, Chroma) was used. A dichroic mirror (T647lpxr, Chroma) was used to split fluorescence signals. Then, emission filters (ET580/60m, Chroma and ET690/50m, Chroma) were used to collect fluorescence signals from donor and acceptor vesicles. MetaMorph was used to calculate average fluorescence intensities and background intensities in ROIs.

\section{Specificity of SNARE proteins.}

DiI and DiD embedded liposomes were prepared in the same way as abovementioned. DiI liposomes were then reconstituted with t-SNAREs and v-SNARE to assembly DiI-tvesicles and DiI-v-vesicles, respectively. DiD liposomes were then reconstituted with tSNAREs and v-SNARE to assembly DiD-t-vesicles and DiD-v-vesicles, respectively. $3 \mu \mathrm{M}$ DiI-t-vesicles were incubated with $3 \mu \mathrm{M}$ DiD-t-vesicles for 30 minutes before loading into NeutrAvidin coated chambers. Images were taken after another 10-minutes incubation for vesicles mounting and further washout. Fused vesicles were counted for calculating the fusion rates between t-vesicles and t-vesicles. Fusion rate was defined as the total number of fusions from acquired images and normalized by the imaging area in $\mu \mathrm{m}^{2}$ and lipid concentration in $\mu \mathrm{M}$. Similar measurements were performed with DiI-t-vesicles and DiD-v-vesicles to calculate the fusion rate between t-vesicles and v-vesicles, and with DiI-v-vesicles and DiD-v-vesicles to calculate the fusion rate between v-vesicles and v-vesicles. 


\section{Supporting Figures}

(a)

mClover3-2aa-mRuby3

mClover3-80aa-mRuby3

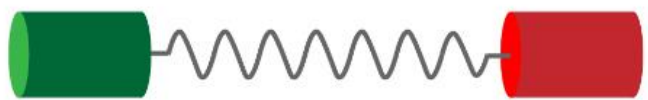

(c)

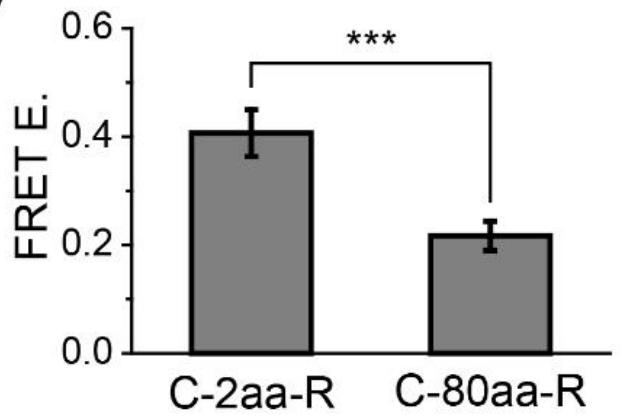

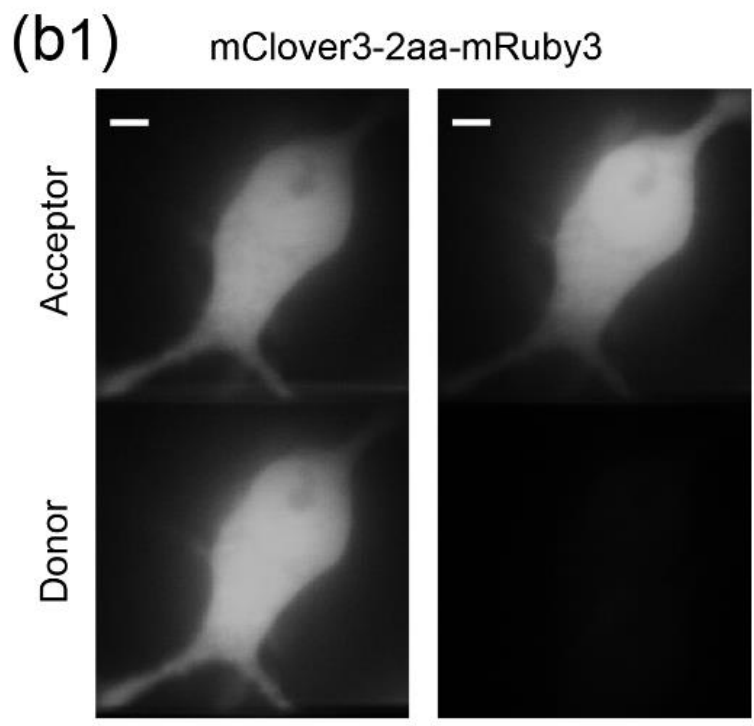

488nm illumination $561 \mathrm{~nm}$ illumination (b2) mClover3-80aa-mRuby3
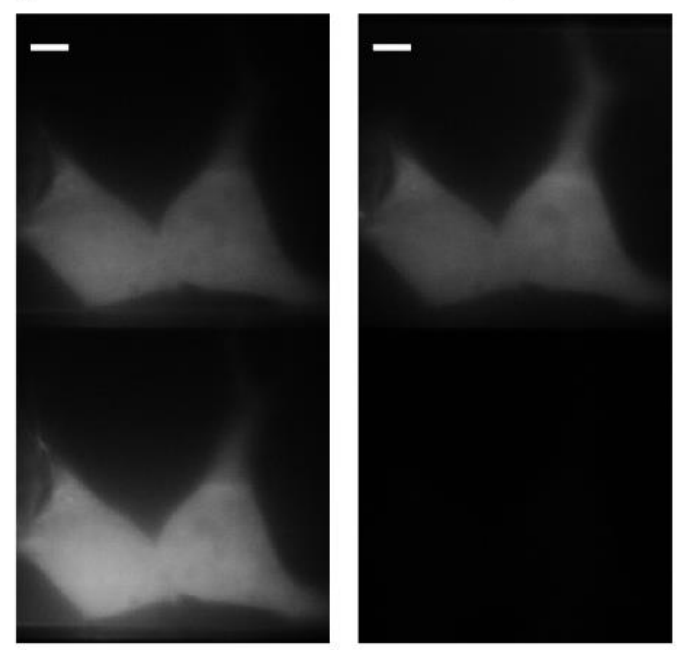

$488 \mathrm{~nm}$ illumination $561 \mathrm{~nm}$ illumination

Figure S1. Measurement of live-cell FRET using a fluorescent protein-based FRET pair in HEK293T cells. (a) Diagrams of mClover3-2aa-mRuby3 and mClover3-80aa-mRuby3 . The linkers of different size ( 2 aa and 80 aa) were connected between mClover3 and mRuby3. (b) Fluorescence images of mClover3-2aa-mRuby3 (b1) and mClover3-80aa-mRuby3 (b2) under the illumination of $488 \mathrm{~nm}$ or $561 \mathrm{~nm}$ lasers in living HEK293T cells. Scale bar: $5 \mu \mathrm{m}$. (c) Live-cell FRET efficiency of mClover3-2aa-mRuby3 (C-2aa-R) and mClover3-80aa-mRuby3 (C-80aa-R) in HEK293T cells. FRET efficiency of mClover3-2aa-mRuby3 $(0.41 \pm 0.043$ (mean \pm standard error of the mean $(\mathrm{SEM}), \mathrm{N}=19)$ ) is higher than that of mClover3-80aamRuby3 $(0.22 \pm 0.027(\mathrm{~N}=19))$. ***, $p<0.001$ (Student's $t$-test) 
(a1)

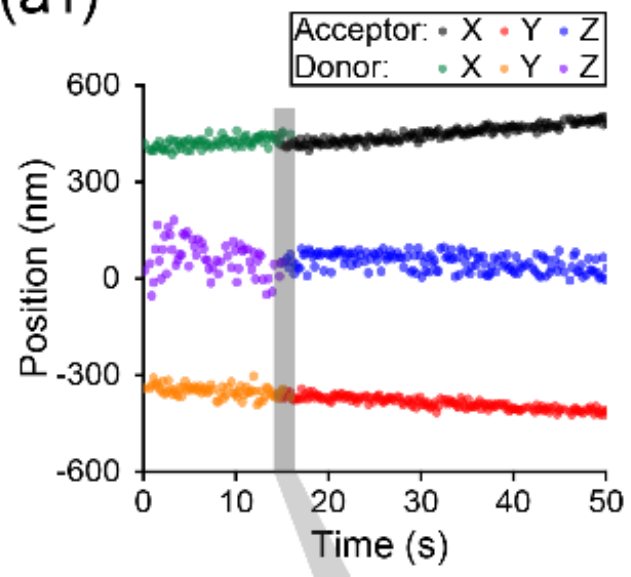

(a2)

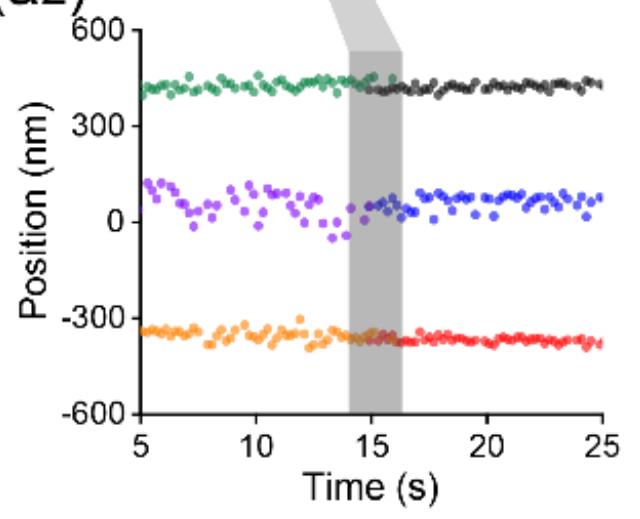

(b1)

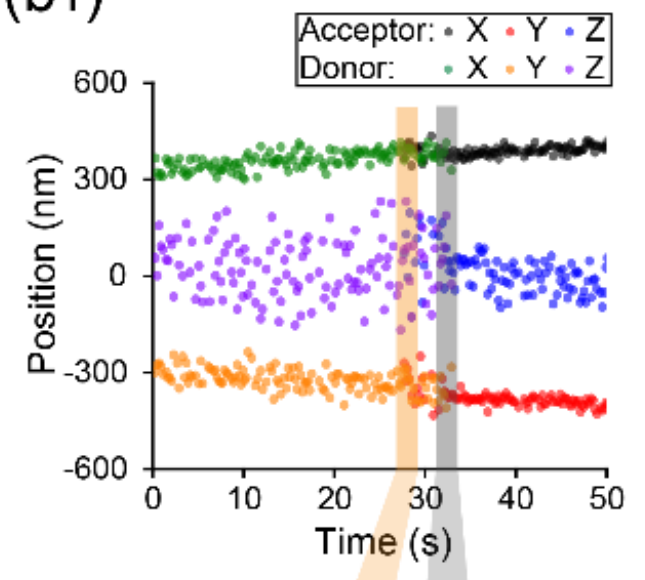

(b2)

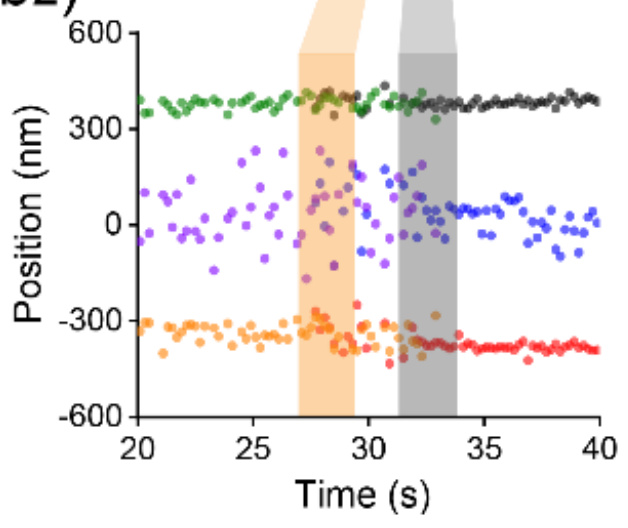

Figure S2. Combined three-dimensional trajectories of DiI-labeled and DiD-labeled vesicles during fusion (a1) and (b1). Three-dimensional trajectories before and after fusion were expanded at (a2) and (b2). Vertical yellow bar: docking. Vertical gray bar: fusion. 

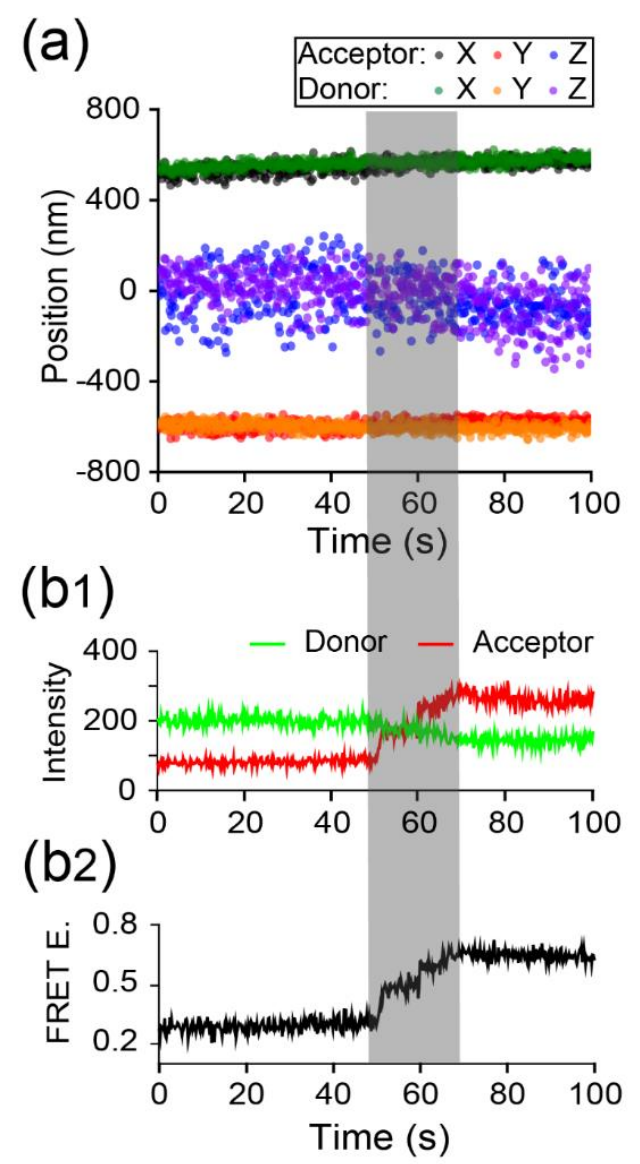

Figure S3. Real-time three-dimensional localization and FRET of a single reconstituted DiDlabeled vesicle and a DiI-labeled vesicle undergoing multiple-step vesicle fusion. (a) Real-time combined three-dimensional trajectories of a DiD-labeled vesicle and a DiI-labeled vesicle in vesicle fusion. (b) Real-time fluorescence intensity time traces (b1) and FRET efficiency trace (b2). Vertical gray bar: multiple-step fusion. 


\section{References}

1. Lee, S. K.; Lee, M. H.; Jeong, S. J.; Qin, X.; Lee, A. R.; Park, H.; Park, C. Y., The inactivation domain of STIM1 acts through intramolecular binding to the coiled-coil domain in the resting state. Journal of cell science 2020, 133 (1).

2. Qin, X.; Liu, L.; Lee, S. K.; Alsina, A.; Liu, T.; Wu, C.; Park, H.; Yu, C.; Kim, H.; Chu, J.; Triller, A.; Tang, B. Z.; Hyeon, C.; Park, C. Y.; Park, H., Increased Confinement and Polydispersity of STIM1 and Orai1 after $\mathrm{Ca}(2+)$ Store Depletion. Biophysical journal 2020, 118 (1), 70-84.

3. Alsina, A.; Lai, W. M.; Wong, W. K.; Qin, X.; Zhang, M.; Park, H., Real-time subpixel-accuracy tracking of single mitochondria in neurons reveals heterogeneous mitochondrial motion. Biochemical and biophysical research communications 2017, 493 (1), 776-782.

4. Park, H.; Hanson, G. T.; Duff, S. R.; Selvin, P. R., Nanometre localization of single ReAsH molecules. Journal of microscopy 2004, 216 (Pt 3), 199-205.

5. Selvin, P. R.; Lougheed, T.; Hoffman, M. T.; Park, H.; Balci, H.; Blehm, B. H.; Toprak, E., In Vitro and In Vivo FIONA and Other Acronyms for Watching Molecular Motors Walk. Single-Molecule Techniques: A Laboratory Manual, Cold Spring Harbor Press, Cold Spring Harbor, NY 2008, 37-71.

6. Goshtasby, A., Image registration by local approximation methods. Image and Vision Computing 1988, 6 (4), 255-261.

7. Churchman, L. S.; Spudich, J. A., Colocalization of fluorescent probes: accurate and precise registration with nanometer resolution. Cold Spring Harbor protocols 2012, 2012 (2), $141-9$.

8. $\quad$ Chandradoss, S. D.; Haagsma, A. C.; Lee, Y. K.; Hwang, J. H.; Nam, J. M.; Joo, C., Surface passivation for single-molecule protein studies. Journal of visualized experiments : JoVE 2014, (86).

9. Andoy, N. M.; Sarkar, S. K.; Wang, Q.; Panda, D.; Benitez, J. J.; Kalininskiy, A.; Chen, P., Single-molecule study of metalloregulator CueR-DNA interactions using engineered Holliday junctions. Biophysical journal 2009, 97 (3), 844-52.

10. Wasserman, M. R.; Alejo, J. L.; Altman, R. B.; Blanchard, S. C., Multiperspective smFRET reveals rate-determining late intermediates of ribosomal translocation. Nature structural \& molecular biology 2016, 23 (4), 333-41.

11. Kim, K. M.; Wijerathne, T.; Hur, J. H.; Kang, U. J.; Kim, I. H.; Kweon, Y. C.; Lee, A. R.; Jeong, S. J.; Lee, S. K.; Lee, Y. Y.; Sim, B. W.; Lee, J. H.; Baig, C.; Kim, S. U.; Chang, K. T.; Lee, K. P.; Park, C. Y., Distinct gating mechanism of SOC channel involving STIM-Orai coupling and an intramolecular interaction of Orai in Caenorhabditis elegans. Proceedings of the National Academy of Sciences of the United States of America 2018, 115 (20), E4623-e4632.

12. Chen, H.; Puhl, H. L., 3rd; Koushik, S. V.; Vogel, S. S.; Ikeda, S. R., Measurement of FRET efficiency and ratio of donor to acceptor concentration in living cells. Biophysical journal 2006, 91 (5), L39-41.

13. Tian, F.; Lin, T. C.; Wang, L.; Chen, S.; Chen, X.; Yiu, P. M.; Tsui, O. K. C.; Chu, J.; Kiang, C. H.; Park, H., Mechanical Responses of Breast Cancer Cells to Substrates of Varying Stiffness Revealed by Single-Cell Measurements. The journal of physical chemistry letters 2020, 11 (18), 7643-7649.

14. Diao, J.; Ishitsuka, Y.; Lee, H.; Joo, C.; Su, Z.; Syed, S.; Shin, Y. K.; Yoon, T. Y.; Ha, T., A single vesicle-vesicle fusion assay for in vitro studies of SNAREs and accessory proteins. Nature protocols 2012, 7 (5), 921-34. 